\title{
A time-delayed feedback technique for suppressing instabilities in time-periodic flow
}

\author{
Léopold ShaAbani Ardali ${ }^{1,2}$, Denis Sipp ${ }^{2}$ and Lutz Lesshafft ${ }^{1}$ \\ ${ }^{1}$ Laboratoire d'Hydrodynamique de l'École polytechnique, \\ École polytechnique - CNRS, \\ Route de Saclay, 91120 Palaiseau, France \\ ${ }^{2}$ Département d'Aérodynamique Fondamentale et Expérimentale, \\ ONERA - Centre de Meudon, \\ 8, rue des Vertugadins, 92190 Meudon, France
}

(Dated: September 5, 2017)

\begin{abstract}
A numerical method is presented that allows to compute time-periodic flow states, even in the presence of hydrodynamic instabilities. The method is based on filtering non-harmonic components by way of delayed feedback control, as introduced by Pyragas [Phys. Lett. A 170, 421-428 (1992)]. Its use in flow problems is demonstrated here for the case of a periodically forced laminar jet, subject to a subharmonic instability that gives rise to vortex pairing. The optimal choice of the filter gain, which is a free parameter in the stabilization procedure, is investigated in the context of a lowdimensional model problem, and it is shown that this model predicts well the filter performance in the high-dimensional flow system. Vortex pairing in the jet is efficiently suppressed, so that the unstable periodic flow state in response to harmonic forcing is accurately retrieved. The procedure is straightforward to implement inside any standard flow solver. Memory requirements for the delayed feedback control can be significantly reduced by means of time interpolation between checkpoints. Finally, the method is extended for the treatment of periodic problems where the frequency is not known a priori. This procedure is demonstrated for a three-dimensional cubic lid-driven cavity in supercritical conditions.
\end{abstract}

\section{INTRODUCTION}

Any analysis of linear flow instability first requires the definition of an unperturbed basic flow state. An obvious problem is that such flow states, if indeed they are unstable, cannot be recovered as asymptotic solutions by simple time-stepping. In the context of steady flow, several methods exist that allow the computation of unstable steady states. Newton-Raphson iteration 1 1 or recursive projection [2, 3] are efficient in many such configurations, although they may require deep modifications of numerical flow solvers, and their convergence is often problematic. A robust alternative, which furthermore is convenient to integrate into an existing time-stepping simulation code, has been proposed by Åkervik et al. [4] under the name of 'selective frequency damping' (SFD). This technique has since been used for a wide variety of steady flow configurations.

Time-periodic flows constitute a distinct class of instability problems, and interest in the computation of periodic states is furthermore not limited to the purpose of instability analysis. Examples include vortex shedding in shear flows [5], pulsating flow in blood vessels [6], and complex flow in turbomachines [7].

Even when a flow settles into an asymptotically stable time-periodic state in the long-time limit, its computation by time-stepping may be costly if long transient dynamics prevail. This difficulty can be overcome by use of the 'harmonic balance' technique [8, 9], which consists in the simultaneous computation of all or many temporal Fourier components of a given periodicity. A pseudo-time is typically employed in order to make all Fourier components converge. This approach is widely used today both in fundamental and in applied contexts. Several improvements of the method address specific issues: if the fundamental period is not known a priori, a 'gradient-based variable time 
period' algorithm [10 13. allows to identify it as an additional unknown of the problem; if the flow is simultaneously forced at several frequencies, the method can be generalized [14. Some strategies for control and shape optimization have also been devised on this basis [15, 16].

Yet time-periodic flows may sustain hydrodynamic instabilities. In particular, the growth of subharmonic perturbations is observed in many such cases. Prominent examples are the pairing of vortices in shear flows [17] and the parametric subharmonic instability (PSI) of internal waves in stratified media [18. Such instabilities may arise from linear dynamics, tractable in the framework of Floquet theory, or from nonlinear effects, as in the case of PSI. It may be possible to retrieve unstable periodic states through harmonic balance, as long as no harmonics of the fundamental flow frequency are involved in the instability, but to the best of our knowledge, this has never been attempted. Shooting methods have been designed to this effect [19, 20, and these have been used successfully in the context of some flow problems [21, 22. However, their implementation requires a considerable overhead around a given flow solver.

The objective of this study is to present an easy-to-implement filtering technique, similar in spirit to the SFD method [4] used for steady flow, that allows the exact computation of time-periodic orbits in stable as well as unstable situations. To this end, an artificial forcing is added to the Navier-Stokes equations, which is required to leave the dynamics of the fundamental flow frequency and of all its higher harmonics unaffected, such that the simulation converges in time towards a periodic solution of the unforced equations. A delayed feedback control [23] achieves this objective. Such time-delay filters have been extensively used in the context of controlling chaotic dynamics in systems with a low number of degrees of freedom. In a recent study [24], a similar technique is applied in a high-dimensional flow problem, in order to suppress spatio-temporal asymmetries in wakes. In the present paper, the use of time-delayed feedback for flow stabilization is explored.

The phenomenon of vortex pairing in an axisymmetrically forced jet is chosen to illustrate the procedure. It is demonstrated how the artificial damping efficiently suppresses the growth of subharmonic perturbations, and thereby the onset of vortex pairing, so that unstable periodic solutions of the Navier-Stokes equations can be obtained. The feedback optimally eliminates subharmonic components, letting the fundamental and its harmonic components unaffected, while all non-harmonic frequencies experience damping. We will show that in weakly stable settings, the feedback can be used to accelerate the convergence towards the asymptotic state. However, this method, due to the full period storage, can be memory-consuming; to severely reduce the memory requirements, we will show how spline interpolation between checkpoints in time can be used, without affecting much the convergence properties of the algorithm.

When flow periodicity arises from intrinsic dynamics, as opposed to external forcing, the period length of the asymptotic state is not known a priori. We will show that the stabilization method for such cases can be extended to identify the period length through iterative adjustment, as will be demonstrated for a cubic lid-driven cavity. Due to their broad range of application, cavities are wellstudied flow systems, which can sustain several types of instabilities 25. A configuration is chosen that is known to give rise to co-existing limit-cycles and intermittently chaotic dynamics [26- 28].

The paper is organized as follows. The jet flow example is introduced in Section 2, and the occurrence of vortex pairing in the absence of artificial damping is discussed. The stabilization method is presented in Section 3. A single free parameter needs to be chosen; its optimal value is found in the context of a simple model problem. Section 4 documents the performance of the technique for an unstable vortex street, with a discussion of the optimal parameter choice. It is further shown how the same technique accelerates the convergence in stable situations, and how the memory requirements may be reduced through check-pointing and interpolation. Details on the simulation technique are provided here. Section 5 extends the stabilization procedure to periodic flows with an unknown period. 


\section{AN EXAMPLE OF SUBHARMONIC INSTABILITY: VORTEX PAIRING IN JETS}

Axisymmetric harmonic forcing at the nozzle of a laminar round jet excites, over a wide range of frequencies, a shear instability of the steady flow state, leading to exponential growth of the perturbation amplitude along the axial direction. As the amplitude reaches nonlinear levels, the shear layer rolls up into a regular street of vortex rings, which form and convect at the frequency of the applied forcing. Depending on flow parameters and forcing frequency (more details given in Sec. IV A), these vortices may undergo subsequent pairing [29], and if the ambient flow is sufficiently quiet and the harmonic forcing is well-controlled, this pairing takes place in a perfectly regular fashion. The numerical method is detailed in Sec. IVA.

In cases where pairing occurs, two neighboring vortices merge into one, such that the passage frequency of vortices downstream of the pairing location is exactly half that of the imposed forcing. If the forcing is characterized by the time period $T$, such that $\omega_{f}=2 \pi / T$, the 'paired state' is globally $2 T$-periodic ( $T$-periodic upstream of the pairing and $2 T$-periodic downstream). The velocity field of a paired state will be denoted $\mathbf{u}^{p}$. An example, obtained by direct numerical simulation, is shown in Fig. 1 a.

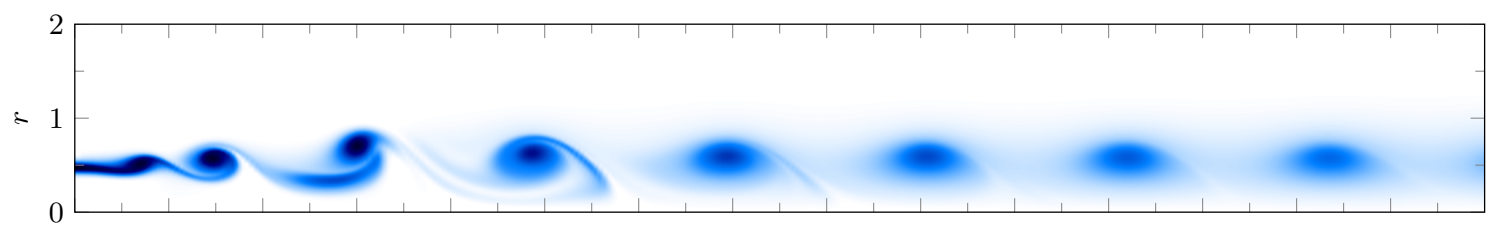

(a) Paired state at $S t=0.6$ and $R e=2000$.

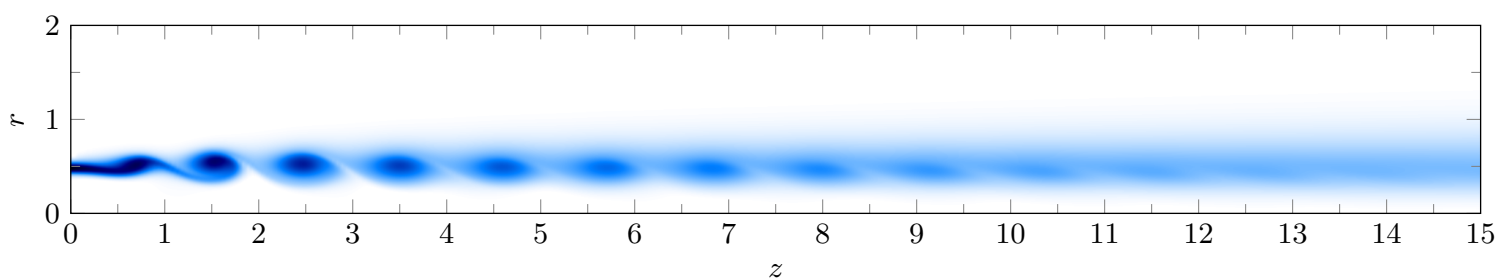

(b) Unpaired state at $S t=0.6$ and $R e=1300$.

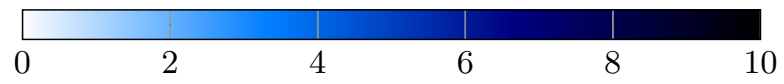

FIG. 1: Vorticity snapshots of paired and unpaired states, obtained without stabilization for two different parameter settings. Reynolds and Strouhal numbers are defined in Sec. IV A.

Another case at different parameter settings, where no pairing is found to occur, is shown in Fig. 1b. Vortices roll up close to the nozzle and advect downstream, until they are dissipated by viscosity. Such a flow state is (globally) $T$-periodic and will be called hereafter an 'unpaired state'. Its velocity field will be denoted $\mathbf{u}^{u}$.

The purpose of this study is to show how, for each paired state, a corresponding unpaired state can be recovered, defining two valid periodic solutions of the Navier-Stokes equations at the same parameter setting. 


\section{SUBHARMONIC STABILIZATION}

In this section, after a brief presentation of filtering techniques (Sec. III A), a simple model problem is introduced in order to determine the coefficients of a time-delayed feedback - here an additional term added to the momentum equation — so that the forced Navier-Stokes simulation converges towards a $T$-periodic state.

\section{A. Time-delayed feedback}

A fully synchronized paired state can be decomposed into components that are $T$-periodic and those that are only $2 T$-periodic,

$$
\begin{aligned}
\mathbf{u}^{p}(\mathbf{x}, t) & =\sum_{n} \mathbf{u}_{n}^{T}(\mathbf{x}) \exp \left(\mathrm{i} n \omega_{f} t\right)+\sum_{n} \mathbf{u}_{n}^{2 T}(\mathbf{x}) \exp \left(\mathrm{i} \frac{2 n+1}{2} \omega_{f} t\right), \\
\text { with } & n=0, \pm 1, \pm 2, \ldots, \pm N .
\end{aligned}
$$

An unpaired state, in contrast, is purely $T$-periodic,

$$
\mathbf{u}^{u}(\mathbf{x}, t)=\sum_{n} \mathbf{u}_{n}^{T}(\mathbf{x}) \exp \left(\mathrm{in} \omega_{f} t\right)
$$

The objective is to design a filter that will damp all $2 T$-periodic components under the second sum in 1a), while leaving any $T$-periodic flow state unaffected. Of course, this filter should also lead to a stable global system.

A first approach might be to consider a standard linear band-stop filter $H$ that cuts around the subharmonic frequency $\omega_{f} / 2$ (i.e. gain $\left|H\left(\omega_{f} / 2\right)\right| \ll 1$ ), while preserving the steady component and the fundamental frequency $\left(H(0)=H\left(\omega_{f}\right)=1\right)$. However, in order to achieve such characteristics, a very high-order filter is needed: in logarithmic scale, $\omega_{f} / 2$ and $\omega_{f}$ are apart by only $\log (2)=0.69$, whereas the gains are separated by $-\log \left(\left|H\left(\omega_{f} / 2\right)\right|\right) \gg 1$. This filter would be cumbersome to implement, and it would require a careful stability and pole placement analysis, as described for example by Aström \& Murray [30, or by Doyle et al. 31. Furthermore, such a filter could not satisfy all requirements: the gain at $\omega_{f} / 2$ cannot be strictly zero, and no constraint can be imposed on the higher $2 T$-periodic harmonics $\left( \pm \frac{3}{2} \omega_{f}, \pm \frac{5}{2} \omega_{f}, \ldots\right)$.

A better approach, that will be adopted here, is to use time-delayed feedback (TDF), as described by Pyragas [23. When the flow at time $t$ is compared with the flow at time $t-T$, components of period $T$ and of period $2 T$ are cleanly distinguished.

The $2 T$-periodic components in a paired state $1 \mathrm{a}$, which are the target of artificial damping, are thus isolated as

$$
\mathbf{u}^{p}(\mathbf{x}, t)-\mathbf{u}^{p}(\mathbf{x}, t-T)=2 \sum_{n} \mathbf{u}_{n}^{2 T}(\mathbf{x}) \exp \left(\mathrm{i} \frac{2 n+1}{2} \omega_{f} t\right),
$$

whereas a $T$-periodic unpaired state satisfies

$$
\mathbf{u}^{u}(\mathbf{x}, t)-\mathbf{u}^{u}(\mathbf{x}, t-T)=0 .
$$

Then, adding a forcing term of the form

$$
\mathbf{f}=-\lambda(\mathbf{u}(t)-\mathbf{u}(t-T))
$$




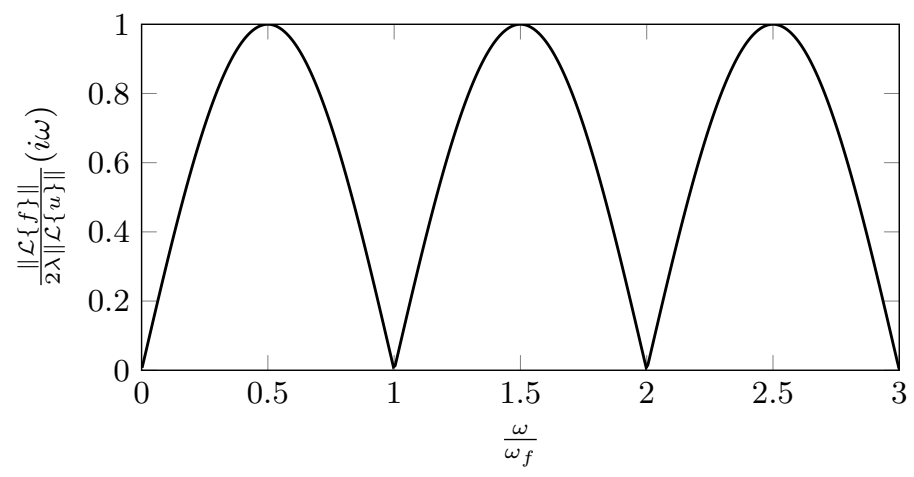

FIG. 2: Gain of the delayed feedback transfer function.

to the right-hand side of 12 allows to control $2 T$-periodic fluctuations without any forcing on $T$-periodic dynamics. In this framework, $\lambda$ is a forcing parameter that needs to be prescribed (see Sec. III B].

The Laplace transform of this forcing term is

$$
\mathcal{L}\{f\}=-\lambda\left(1-e^{-\omega T}\right) \mathcal{L}\{u\},
$$

so that its gain for a given frequency $\omega$ is found as

$$
\frac{\|\mathcal{L}\{f\}\|}{\|\mathcal{L}\{u\}\|}(i \omega)=\lambda \sqrt{2-2 \cos (\omega T)} .
$$

The resulting transfer function is plotted in Fig. 2, The time-delayed feedback damps all frequencies that are not harmonics of $\omega_{f}$, with maximum effect on the subharmonic frequency $\omega_{f} / 2$ and on its odd harmonics $(n+1 / 2) \omega_{f}$. It is neutral with respect to the mean flow, the fundamental frequency $\omega_{f}$ and its harmonics $n \omega_{f}$.

If the forced system converges towards a $T$-periodic unpaired state, the forcing will vanish, such that the recovered state is a consistent solution of the unforced Navier-Stokes equations.

\section{B. Choice of the feedback parameter $\lambda$}

At first glance, it might be expected from (6) that larger values of $\lambda$ will always lead to more efficient non-harmonic damping. This however is not the case, similar to what has been demonstrated in the context of low-dimensional chaotic systems [23].

In order to guide the choice of the feedback parameter $\lambda$ for the present purpose, a model problem is proposed. The dynamics of a two-frequency oscillator is considered,

$$
\frac{\mathrm{d}}{\mathrm{d} t}\left(\begin{array}{c}
x^{s} \\
\tilde{x}^{s} \\
x^{h} \\
\tilde{x}^{h}
\end{array}\right)=\left(\begin{array}{cccc}
0 & \frac{\omega_{f}}{2} & 0 & 0 \\
-\frac{\omega_{f}}{2} & 0 & 0 & 0 \\
0 & 0 & 0 & \omega_{f} \\
0 & 0 & -\omega_{f} & 0
\end{array}\right)\left(\begin{array}{c}
x^{s} \\
\tilde{x}^{s} \\
x^{h} \\
\tilde{x}^{h}
\end{array}\right)-\lambda\left(\begin{array}{c}
x^{s}(t)-x^{s}(t-T) \\
\tilde{x}^{s}(t)-\tilde{x}^{s}(t-T) \\
x^{h}(t)-x^{h}(t-T) \\
\tilde{x}^{h}(t)-\tilde{x}^{h}(t-T)
\end{array}\right)
$$


with $T=2 \pi / \omega_{f}$ the period of the fundamental mode. Unlike the flow problem, the two frequencies $\omega_{f}$ and $\frac{1}{2} \omega_{f}$ in this model are uncoupled. After nondimensionalization, $\omega_{f} t \rightarrow t$ and $\lambda / \omega_{f} \rightarrow \lambda$, the system can be diagonalized as

$$
\frac{\mathrm{d}}{\mathrm{d} t}\left(\begin{array}{l}
y^{s} \\
\tilde{y}^{s} \\
y^{h} \\
\tilde{y}^{h}
\end{array}\right)=\left(\begin{array}{cccc}
\frac{i}{2} & 0 & 0 & 0 \\
0 & -\frac{i}{2} & 0 & 0 \\
0 & 0 & i & 0 \\
0 & 0 & 0 & -i
\end{array}\right)\left(\begin{array}{l}
y^{s} \\
\tilde{y}^{s} \\
y^{h} \\
\tilde{y}^{h}
\end{array}\right)-\lambda\left(\begin{array}{l}
y^{s}(t)-y^{s}(t-2 \pi) \\
\tilde{y}^{s}(t)-\tilde{y}^{s}(t-2 \pi) \\
y^{h}(t)-y^{h}(t-2 \pi) \\
\tilde{y}^{h}(t)-\tilde{y}^{h}(t-2 \pi)
\end{array}\right) .
$$

In a general linear problem with time-delayed feedback, the eigenvalues are not found in closed form, and their number is infinite [32. In contrast, exact eigensolutions of the uncoupled problem (8) can be found analytically. Introducing exponential modes, the following system is obtained:

$$
\begin{array}{ccc}
y^{s} \propto e^{\alpha^{s} t} & \Rightarrow \quad \alpha^{s}=\frac{i}{2}-\lambda\left(1-e^{-2 \pi \alpha^{s}}\right), \\
\tilde{y}^{s} \propto e^{\tilde{\alpha}^{s} t} & \Rightarrow \quad \tilde{\alpha}^{s}=-\frac{i}{2}-\lambda\left(1-e^{-2 \pi \tilde{\alpha}^{s}}\right), \\
y^{h} \propto e^{\alpha^{h} t} & \Rightarrow \quad \alpha^{h}=i-\lambda\left(1-e^{-2 \pi \alpha^{h}}\right), \\
\tilde{y}^{h} \propto e^{\tilde{\alpha}^{h} t} & \Rightarrow \quad \tilde{\alpha}^{h}=-i-\lambda\left(1-e^{-2 \pi \tilde{\alpha}^{h}}\right) .
\end{array}
$$

As long as real values are chosen for $\lambda$, the solutions of equations (9a $9 \mathrm{~d}$ ) come in complex conjugate pairs, $\tilde{\alpha}^{s}=\bar{\alpha}^{s}$ and $\tilde{\alpha}^{h}=\bar{\alpha}^{h}$. It is therefore sufficient to consider equations $9 \mathrm{9a}$ 9c and their closedform solutions

$$
\begin{aligned}
\alpha_{j}^{s} & =\frac{i}{2}-\lambda+\frac{1}{2 \pi} W_{j}\left(-2 \pi \lambda e^{2 \pi \lambda}\right), \\
\alpha_{j}^{h} & =i-\lambda+\frac{1}{2 \pi} W_{j}\left(2 \pi \lambda e^{2 \pi \lambda}\right), \quad j \in \mathbb{Z} .
\end{aligned}
$$

$W_{j}$ denotes the $j^{\text {th }}$ branch of the Lambert $W$ function, which is the inverse relation of the complex function $z \mapsto z e^{z}$ [33]. An infinite number of solutions (9a 9c) exist, corresponding to individual branches of the Lambert function. In particular, $W_{0}$ gives $\alpha_{0}^{n}=i$ for any value of $\lambda$, preserving the harmonic dynamics. For the purpose of flow stabilization, only the real part of the $\alpha$ values is of interest, as these govern the growth or decay of fluctuations. If, for a given $\lambda$, there exists at least one $j$ such that the real part of $\alpha_{j}^{s}$ or of $\alpha_{j}^{h}$ is positive, then the system is unstable. Therefore, $\lambda$ must meet two criteria:

1. It should provide the most efficient damping in the subharmonic component equation (9a). For a given $\lambda$, it is always sufficient to consider the least stable mode among all possible solutions, i.e. the mode $\alpha_{j}^{s}$ with the largest real part in equation 10a. The optimal value of $\lambda$ leads to maximal decay in the least stable mode.

2. At the same time, $\lambda$ must not create any instability in the fundamental equation (9c); the real part of $\alpha_{j}^{h}$ must be negative for every $j \in \mathbb{Z}$. While the neutral fundamental mode $\alpha_{0}^{h}=i$ exists irrespective of $\lambda$, it must not be dominated by any unstable mode.

In order to identify the optimal $\lambda$ according to these requirements, the following result is demonstrated in appendix if, for a given value of $\lambda$, equations (9a) or 9c have unstable solutions, the branch $j$ on which this solution lies is such that

$$
|j|<2 \lambda+1
$$


As will be seen later, optimal subharmonic damping is found to be achieved within the range $0<\lambda<2$; consequently, the stability of the fundamental component must be ascertained for this range of $\lambda$, and the branches $-4 \leq j \leq 4$ are to be considered.

Figure 3 shows that no fundamental modes on these branches are unstable for any value of $\lambda$. As expected, the neutral eigenvalue $\alpha_{0}^{h}=i$ is always recovered, which is consistent with the premise that the applied forcing does not modify the fundamental dynamics. Therefore, the stability requirement for the fundamental modes (criterion 2) does not restrict the choice of $\lambda$.

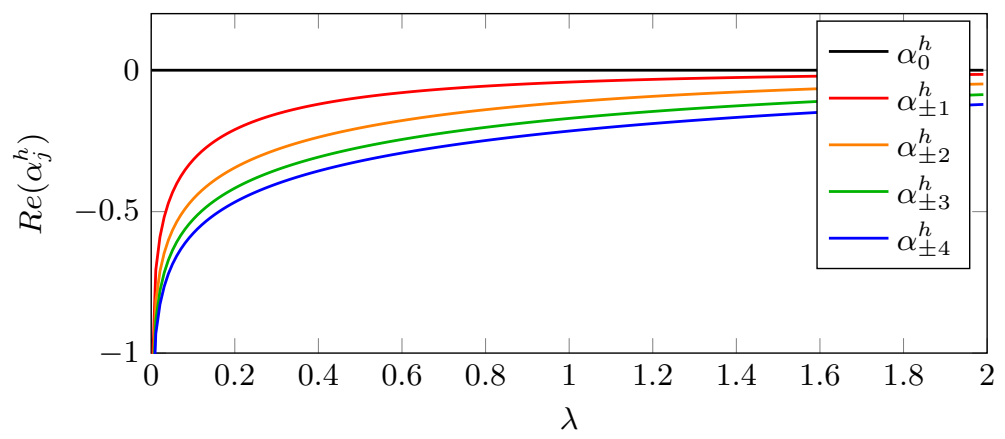

FIG. 3: Real part of eigenvalues $\alpha_{j}^{h}$ for $-4 \leq j \leq 4$, pertaining to fundamental oscillations, as functions of $\lambda$. It is found numerically that $\alpha_{j}^{h}$ and $\alpha_{-j}^{h}$ always have the same real part. The system is neutrally stable for any value of $\lambda$, with the neutral mode $\alpha_{0}^{h}=i$.

Figure 4 demonstrates that the subharmonic modes on branches $-2 \leq j \leq 2$ experience damping for any value of $\lambda$. The same is observed for branches $|j|=3,4$. Therefore, all the subharmonic modes are stable. The least stable modes among these correspond to $j=0$ and $j=-1$. The real parts of $\alpha_{0}^{s}$ and $\alpha_{-1}^{s}$ are identical for $\lambda>0.04432$. This is identified as the optimal $\lambda$ value, as it provides the strongest stabilization of $\alpha_{0}^{s}$.
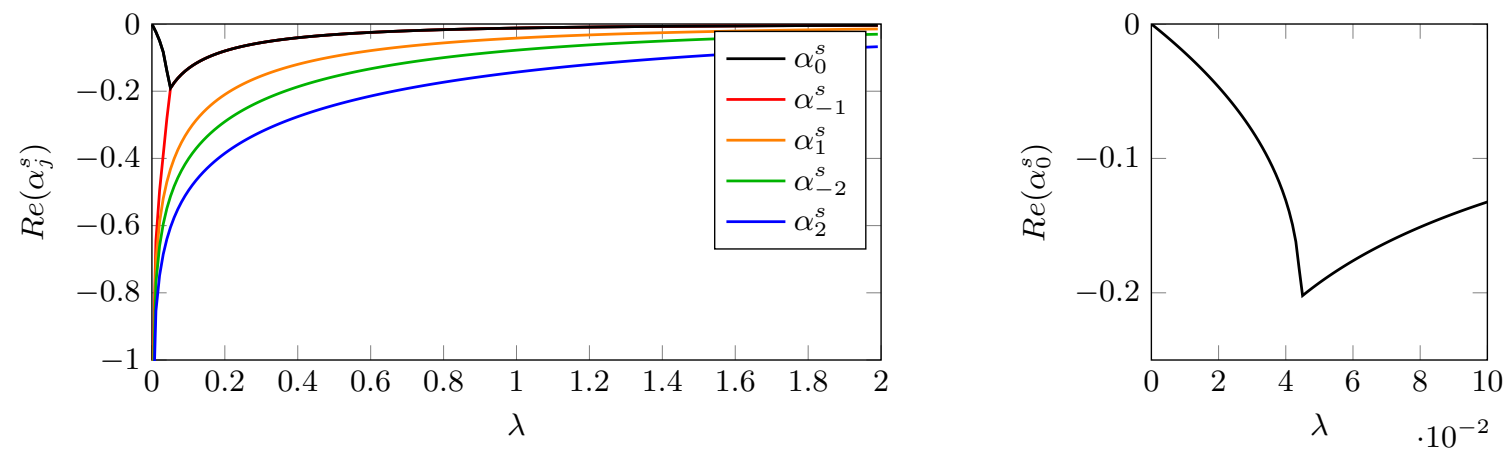

FIG. 4: Real part of eigenvalues $\alpha_{j}^{s}$, pertaining to subharmonic oscillations, as functions of $\lambda$. On the left, eigenvalues for $-2 \leq j \leq 2$; on the right, zoom on small $\lambda$ values only for $j=0$. All these eigenvalues are stable, but least so for the $j=0$ branch. Higher eigenvalues than those shown are even more stable.

It is now examined whether the damped value $\alpha_{0}^{s}=-0.203+0.501 i$ is still the least stable across all $j$ branches. It is demonstrated in the appendix $\llbracket$ that, if such a mode exists, it must stem from a 
branch $j$ such that $|j|<1+\left(1+e^{2 \pi 0.203}\right) \lambda$. For $\lambda<2$, this criterion restricts the search interval to $-10 \leq j \leq 10$. It can be reported that $\alpha_{0}^{s}$ is indeed the least stable eigenvalue of the stabilized system. Consequently, $\lambda=0.04432$ is the optimal value of the damping parameter, leading to a system where the maximum subharmonic growth rate is -0.203 .

\section{STABILIZED VORTEX STREET}

In this section, the TDF technique presented in Sec. III is applied to the case of vortex pairing. The configuration and the numerical code used in this article (Sec. III A) is first described in some more detail. Then, in Sec. IVB, it is demonstrated that adding a time-delayed feedback makes a Navier-Stokes simulation converge towards the unstable unpaired state when initialized with the natural paired state. In Sec. IVC, it is confirmed that the simple model problem provides the optimal coefficient in the present vortex pairing case. Finally, in Sec. IVD, the technique is shown to also provide an efficient means to accelerate convergence in the case of a stable unpaired state.

\section{A. Simulation Method}

Direct numerical simulations were carried out using NEK5000 [34, an incompressible spectral element code. An axisymmetric laminar jet is described in cylindrical coordinates $(z, r), z$ being the main flow direction and $r$ being the radial distance from the jet axis. The flow is assumed to be governed by the incompressible Navier-Stokes equations with zero azimuthal velocity, written in dimensionless form as

$$
\frac{\partial \mathbf{u}}{\partial t}+(\mathbf{u} \cdot \nabla) \mathbf{u}=-\nabla p+\frac{1}{R e} \Delta \mathbf{u}, \quad \nabla \cdot \mathbf{u}=0 .
$$

The velocity $\mathbf{u}$ has axial and radial components $u$ and $v$, and $p$ denotes pressure. The jet diameter $D$ and the inlet centerline velocity $U_{0}$ are used to render the flow problem nondimensional, defining the Reynolds number as $R e=U_{0} D / \nu$, with $\nu$ the kinematic viscosity. The computational domain extends over $15 \times 5$ diameters in the axial and radial directions, respectively, and it is discretized with 6600 spectral elements, each containing 64 mesh points. Mesh convergence has been validated by comparing results for different spectral polynomial orders $(n=4,6,8$ and 10; 8 being the standard). Boundary conditions are specified as follows.

1. In the inlet plane, $z=0$, a hyperbolic-tangent velocity profile is imposed. In dimensionless form, its amplitude is modulated in time as

$$
\mathbf{u}(r, t)=\frac{1}{2}\left\{1-\tanh \left[\frac{1}{4 \theta_{0}}\left(r-\frac{1}{4 r}\right)\right]\right\}\left(1+A \cos \left(\omega_{f} t\right)\right) \mathbf{e}_{\mathbf{z}},
$$

where $A=0.05$ is the forcing amplitude of the jet, $\theta_{0}=0.025$ is the initial dimensionless mixing layer thickness and $\omega_{f}$ is the axial forcing frequency. The periodic nature of the flow is imposed with the periodic inlet forcing, similar as in Jacobs \& Durbin [35. The forcing period is given by $T=2 \pi / \omega_{f}$, and the Strouhal number is defined as $S t=\omega_{f} D /\left(2 \pi U_{0}\right)$.

2. On the centerline of the jet, $r=0$, axisymmetric boundary conditions are imposed,

$$
\frac{\partial u}{\partial r}=v=\frac{\partial p}{\partial r}=0
$$


3. in the outlet plane, $z=15$, and on the lateral boundary, $r=5$, a stress-free outflow condition is applied:

$$
-p \mathbf{n}+\frac{1}{R e}\left(\nabla \mathbf{u}+\nabla \mathbf{u}^{t}\right) \mathbf{n}=0
$$

The flow configuration is thus characterized by the Reynolds number Re, the Strouhal number $S t$, the dimensionless mixing layer thickness $\theta_{0}$ and the forcing amplitude $A$.

\section{B. Computation of an unstable unpaired state}

The stabilization technique described in Sec. IIIA is now applied, by adding a time-delayed feedback term

$$
\mathbf{f}(t)=-\lambda \omega_{f}(\mathbf{u}(t)-\mathbf{u}(t-T))
$$

to the right-hand side of the Navier-Stokes equations (12). The parameter setting $R e=2000$ and $S t=0.6$ has previously been found to exhibit synchronized vortex pairing in the absence of stabilization (Figure 1a), and will serve as example case. The action of the feedback is measured by tracing a norm of non-harmonic (in the sense of non- $T$-periodic) fluctuations, defined as

$$
e(t)=\frac{1}{2} \sqrt{\int_{z} \int_{r} r\|\mathbf{u}(t)-\mathbf{u}(t-T)\|^{2} \mathrm{~d} r \mathrm{~d} z} .
$$

This quantity measures the residual during the stabilization process.

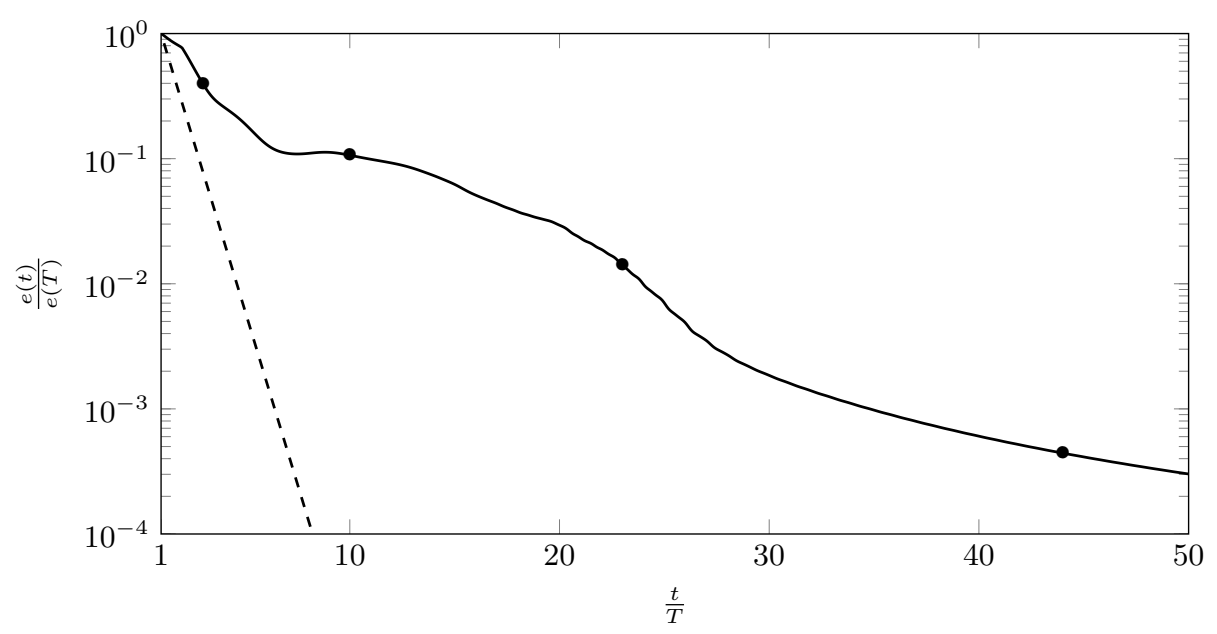

FIG. 5: Evolution of the residual norm $e(t)$ (equation 17), in a jet simulation with $R e=2000$, $S t=0.6$ and $\lambda=0.04432$. Dashed line: decay rate found in the model problem. Markers indicate the instances of snapshots shown in Fig. 6.

The simulation is started at $t=0$ from the paired state represented in Fig. 1 a and the optimal value $\lambda=0.04432$ as identified in Sec. IIIB is used first. Feedback is switched on at $t=T$, because 


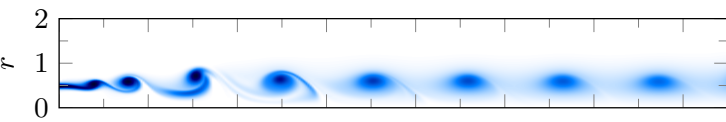

(a) Vorticity at $t=T$.

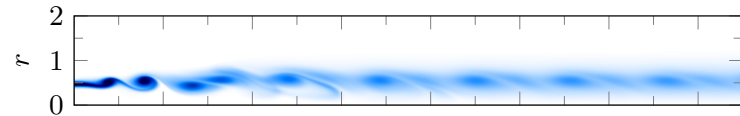

(c) Vorticity at $t=3 T$.

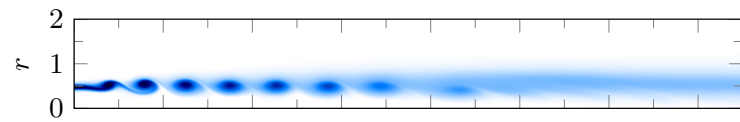

(e) Vorticity at $t=10 T$.

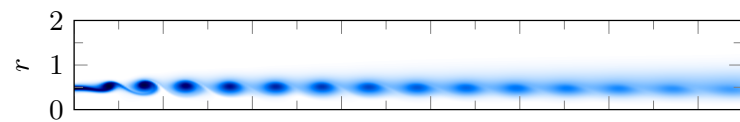

(g) Vorticity at $t=23 T$.

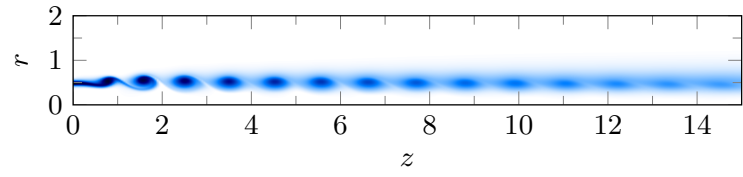

(i) Vorticity at $t=44 T$.

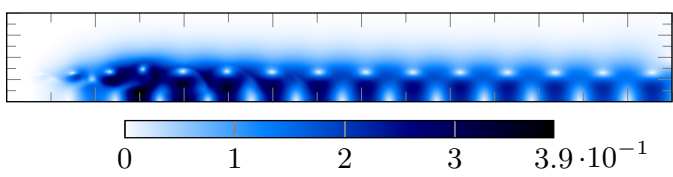

(b) Non-harmonic component at $t=T$.

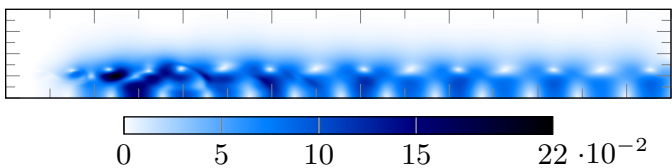

(d) Non-harmonic component at $t=3 T$.

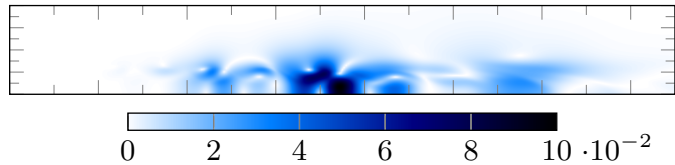

(f) Non-harmonic component at $t=10 \mathrm{~T}$.

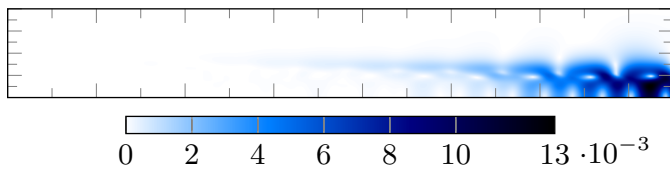

(h) Non-harmonic component at $t=23 T$.

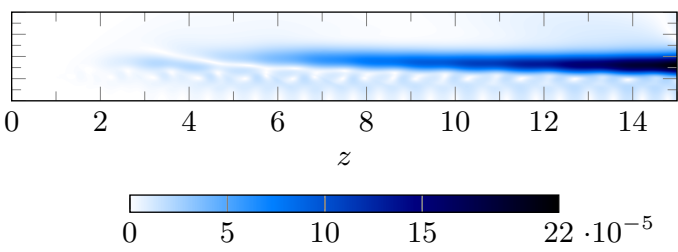

(j) Non-harmonic component at $t=44 T$

FIG. 6: Vorticity and non-harmonic component magnitude $\|\mathbf{u}(t)-\mathbf{u}(t-T)\|$ for $\lambda_{\text {opt }}=0.04432$.

The vorticity colorbar is in Fig. $1 \mathrm{~b}$

one flow period needs to be recorded before the TDF term can be evaluated. The evolution of $e(t)$ is plotted in Fig. 5 f four phases in the stabilization process can be distinguished.

During the first phase, the applied forcing quenches the $2 T$-periodic paired vortices. The distinct vortex structures downstream of the pairing location are thus replaced by a diffuse band of vorticity, as seen by comparing Figs. $6 \mathrm{a}$ and $6 \mathrm{c}$. The magnitude of the non-harmonic component, $\| \mathbf{u}(t)-\mathbf{u}(t-$ $T) \|$, which is proportional to the magnitude of the instantaneous forcing, is displayed in Fig. 6d the forcing at this stage is active in the entire paired region, but not in the region of initial vortex roll-up. This behavior is typical for $0<t<5 T$, when the decay of the non-harmonic component 
is fastest, according to Fig. 5. This stage of the stabilization process is conceptually similar to the subharmonic damping in the model problem of Sec. IIIB However, the damping rate observed in the jet is smaller than predicted by the model. This may be explained by the inherent positive subharmonic growth in the jet, which the damping has to overcome, whereas no such growth was assumed in the model problem.

During the following phase, the flow domain is gradually re-populated by a street of unpaired vortices, essentially by convection, as shown in Fig. 6e. This interpretation is consistent with the map in Fig. 6f], where non-harmonic fluctuations are seen to be concentrated around the trailing end of the emerging vortex array. This behavior dominates the plateau region around $t=10 T$ in Fig. 5

The third phase begins as the unpaired vortex street reaches the downstream end of the domain, when the flow visually appears to have reached a periodic state, displayed in Fig. 6g. The nonharmonic fluctuations at the trailing end of the vortex street leave the domain at this point, as seen in Fig. 6h, and this leads to a second sudden drop in the residual norm $e(t)$ in Fig. 5 .

In the final phase, the flow is globally synchronized, and no visible difference between subsequent periods is observed anymore. Fig. 6i shows the flow state at $t=44 T$. The residual norm continues to slowly decay in time as residual fluctuations are suppressed. These fluctuations are located far from the jet inlet, see Fig. 6j, and they do not present any spatial structure that can be associated with vortex pairing.

\section{Validation of the optimality of the feedback parameter $\lambda$}

In the preceding section, $\lambda$ has been prescribed as the optimal value derived in the context of a model problem. The optimality for the present flow problem is now to be assessed. The simulation from Sec. IVB is repeated, over a time horizon of $250 T$, with sixteen different values of $\lambda$ between 0.01 and 2. The time evolution of $e(t)$ is documented in Figs. $7 \mathrm{a}$ and $7 \mathrm{~b}$ for each value $0.01 \leq \lambda \leq 0.5$. Larger values give poor results and are not reported.

Comparable results are achieved with $0.03 \leq \lambda \leq 0.2$; all curves in this range display the same characteristic phases of convergence, albeit with different efficiencies over short times. The longtime residual $e(t \gg T)$ is seen in Fig. 8 to be insensitive to the choice of $\lambda$ within these limits. However, an optimal $\lambda$ value may be identified that induces the fastest convergence towards the final phase, i.e. the $\lambda$ for which the end of the third phase defined in IVB is reached in the shortest time. Figs. $7 \mathrm{a}$ and $7 \mathrm{~b}$ show that the optimal value in this sense, among all values tried, is indeed $\lambda=0.04432$, the one obtained in Sec. IIIB.

\section{Convergence acceleration in a stable setting}

In the context of steady flows, selective frequency damping is effective in stabilizing unstable settings, but it also provides accelerated convergence towards a steady state in weakly stable situations [4. Time-delayed feedback may achieve the same for weakly stable periodic flow. The case of a jet at $R e=1300$, forced at $S t=0.6$, is chosen for a demonstration. The stable periodic solution in this setting is the unpaired state presented in Fig. 1b. This case is close to the onset of a pairing instability, as the same configuration with $R e=1400$ settles into a stable paired state. Convergence of the final periodic unpaired state at $R e=1300$ is slow as a consequence.

A converged steady laminar state without inflow forcing is chosen as initial condition, and harmonic inflow forcing 13 is started at $t=0$. Simulations are then performed with and without 


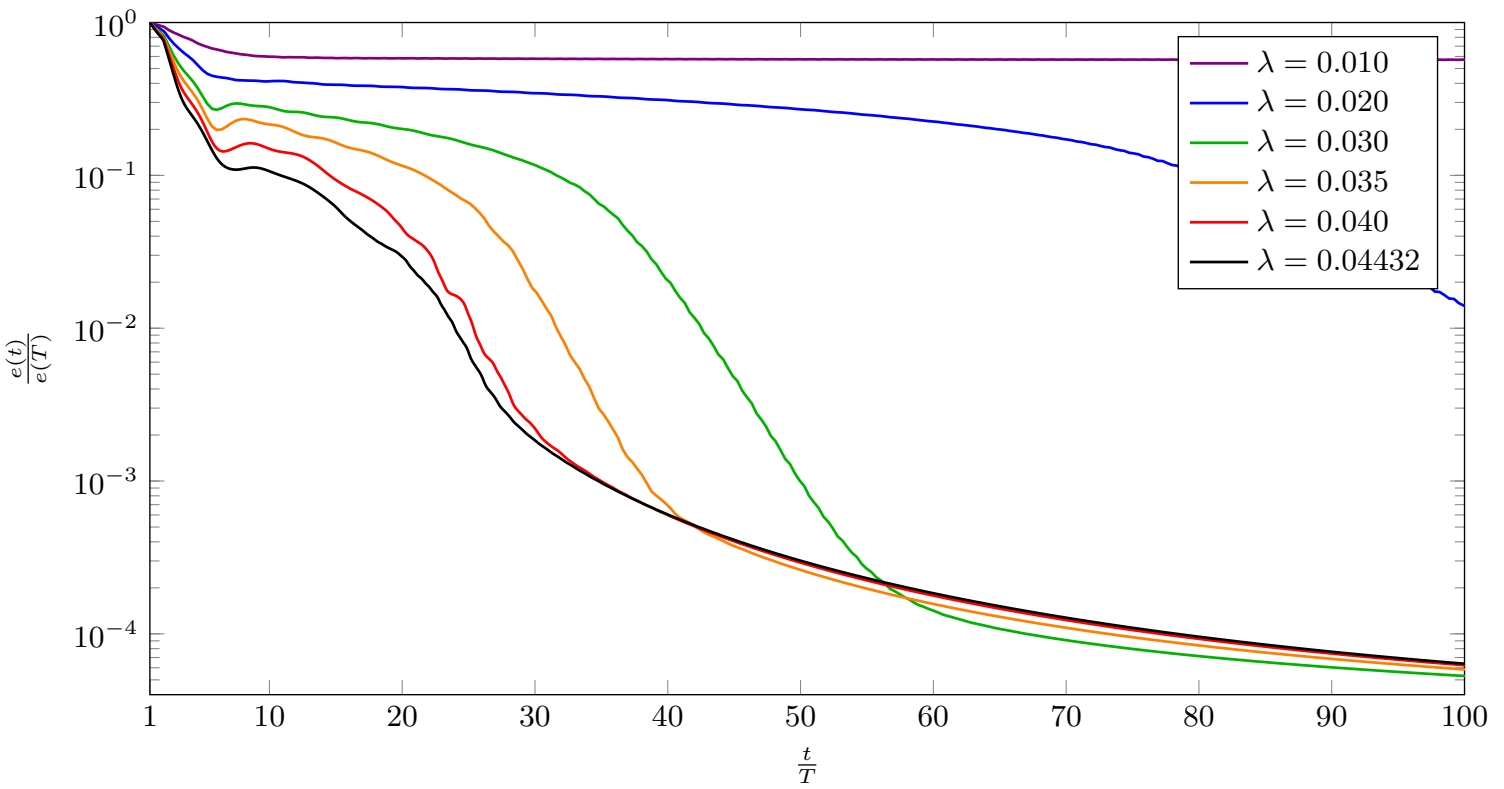

(a) Here, $\lambda \leq 0.0432$.

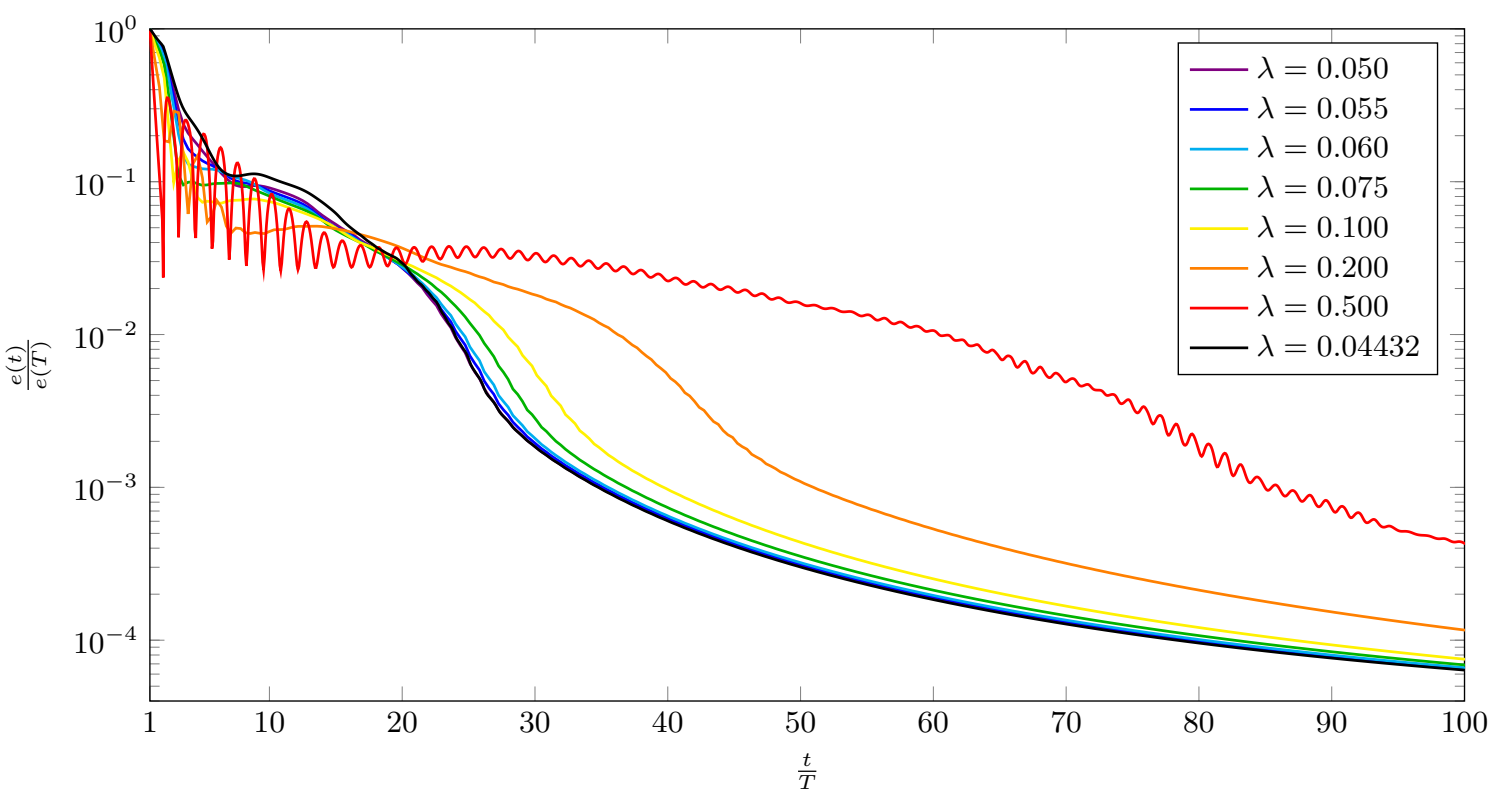

(b) Here, $\lambda \geq 0.0432$.

FIG. 7: Residual norm as a function of time for several values of $\lambda$. Curves for $\lambda=0.0425,0.0475$ are omitted for clarity. At values $\lambda>0.5$, the convergence is increasingly ill-behaved, displaying huge oscillating behavior, and results are not reported. 


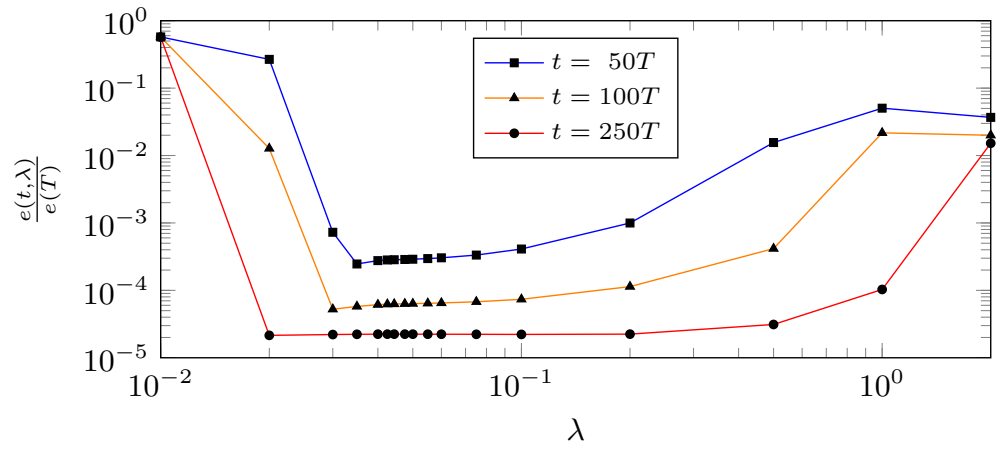

FIG. 8: Residual norm $e(t)$ at $t=50 T, 100 T$ and $250 T$ as a function of $\lambda$.

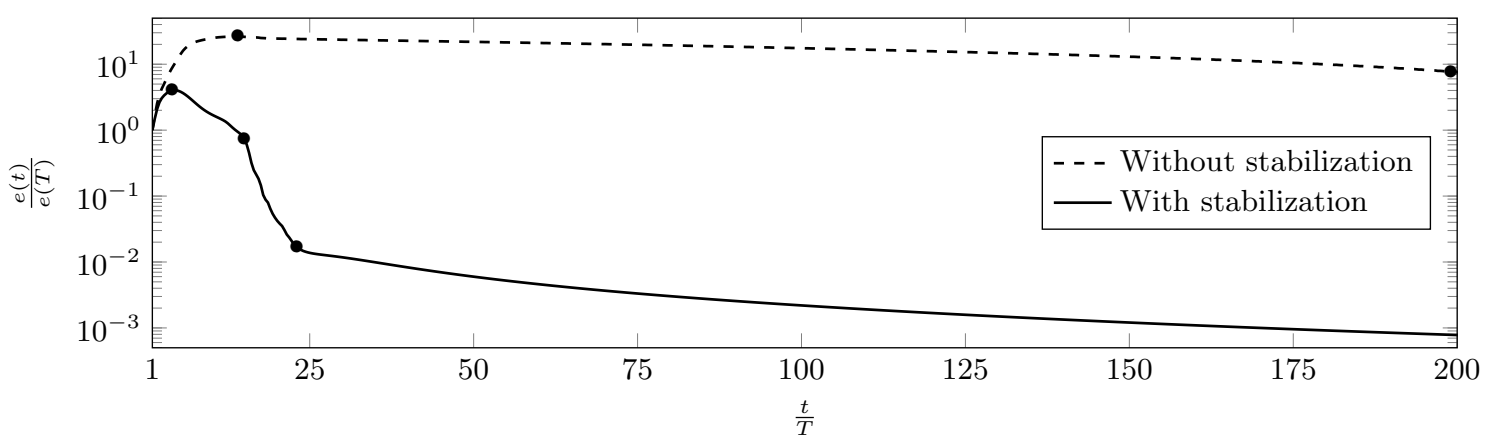

FIG. 9: Evolution of the residual norm $e(t)$ with and without stabilization applied. The different time-steps defined in Sec. IV D are reported.

time-delayed feedback; the non-harmonic component norm $e(t)$ is plotted as a function of time for both runs in Fig. 9

Without damping, pairing sets in quickly several diameters downstream of the inlet. The paired vortex is then convected downstream, while repeated pairing takes place at almost the same location, such that the global norm of non- $T$-periodic components continue to grow (dashed line in Fig. 9). This growth ends at $t=14 T$, when the first paired ring reaches the outlet, as can be seen in Figs. 10a and 10b. Subsequently, $e(t)$ decays as the pairing location moves slowly downstream. At the end of the simulation, at $t=200 T$, pairing still takes place near the downstream end of the domain, as shown in Figs. $10 \mathrm{c}$ and 10d, Evacuation of the transient pairing through convection is a very slow process in this setting.

In the presence of time-delay feedback, pairing is never observed, and the convergence is significantly accelerated. According to Fig. 9, subharmonic fluctuations are reduced to residual levels within 20 forcing periods, which corresponds to the convection time of vortices through the domain. Snapshots of vorticity and of non-harmonic components are shown in Fig. 11 for three notable instances, as marked in Fig. 9 . 


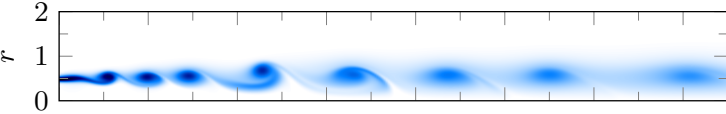

(a) Vorticity at $t=14 T$.

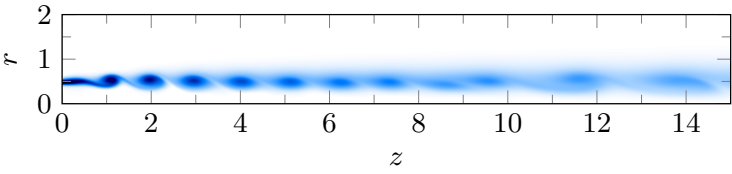

(c) Vorticity at $t=200 T$.

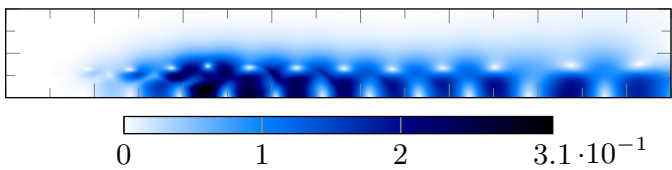

(b) Non-harmonic component at $t=14 T$.

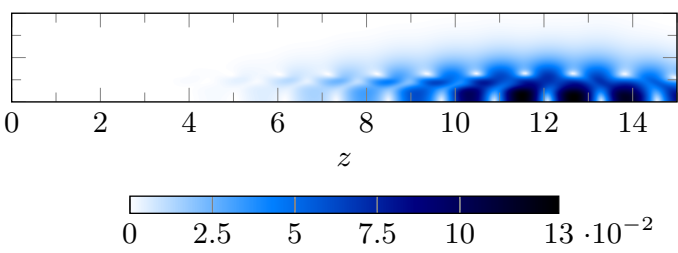

(d) Non-harmonic component at $t=200 T$.

FIG. 10: Vorticity and non-harmonic component magnitude $\|\mathbf{u}(t)-\mathbf{u}(t-T)\|$ without feedback applied. The vorticity colorbar is in Fig. $1 \mathrm{~b}$.

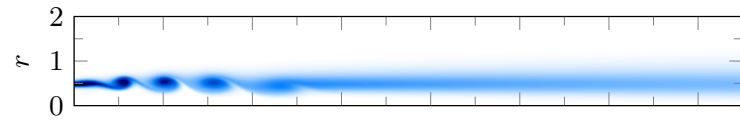

(a) Vorticity at $t=4 T$.

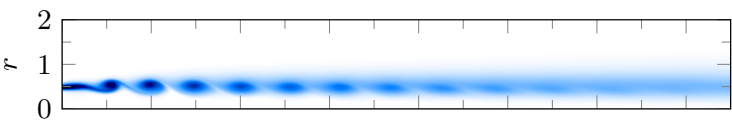

(c) Vorticity at $t=15 T$.

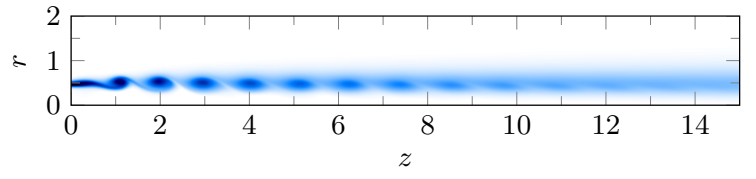

(e) Vorticity at $t=23 T$.

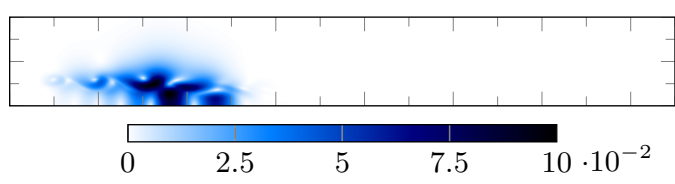

(b) Non-harmonic component at $t=4 T$.

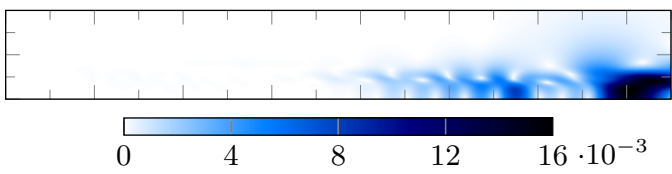

(d) Non-harmonic component at $t=15 T$.
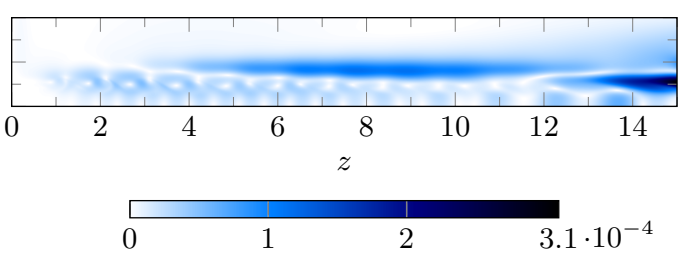

(f) Non-harmonic component at $t=23 T$.

FIG. 11: Vorticity and non-harmonic component magnitude $\|\mathbf{u}(t)-\mathbf{u}(t-T)\|$ with time-delayed feedback applied. The vorticity colorbar is in Fig. 1b. 


\section{E. Reducing the memory requirements through time interpolation}

The TDF method described so far, although easy to implement, needs the storage of one full flow period, which can be resource-intensive, especially in the case of three-dimensional simulations. A remedy may be to store all flow variables and their time-derivatives only at $N$ equispaced instants over one period, and to approximate all intermediate time steps through interpolation.

A first interpolation technique could rely on Fourier methods, since the converged flow is $T$ periodic. However, since the algorithm is based on the damping of non-periodic components, accurate reconstruction of these component precludes the use of Fourier series.

A spline interpolation is tried instead: each period is composed of $N_{\Delta t}$ time steps, and $N$ equispaced time steps of the previous running period are stored in memory, i.e. one time step every $N_{\Delta t} / N$ time steps. The time derivative $\mathbf{u}_{t}$ of the velocity at each time step, computed with a centered-difference scheme, is also stored. Then, to reconstruct the flow at $t-T$, if $t_{i} \leq t-T \leq t_{i+1}$, with $t_{i}$ and $t_{i+1}$ time steps where the flow is stored, the following spline interpolation formula is used:

$$
\begin{array}{r}
\tilde{\mathbf{u}}(r, z, t-T)=\left(1-t^{\prime}\right)^{2}\left(1+2 t^{\prime}\right) \mathbf{u}\left(r, z, t_{i}\right)+t^{\prime}\left(1-t^{\prime}\right)^{2} \frac{T}{N} \mathbf{u}_{t}\left(r, z, t_{i}\right)+ \\
t^{\prime 2}\left(3-2 t^{\prime}\right) \mathbf{u}\left(r, z, t_{i+1}\right)+t^{\prime 2}\left(t^{\prime}-1\right) \frac{T}{N} \mathbf{u}_{t}\left(r, z, t_{i+1}\right),
\end{array}
$$

with the normalized time

$$
t^{\prime}=\frac{t-T-t_{i}}{t_{i+1}-t_{i}}
$$

This interpolation technique yields interpolated values, continuous up to the first time-derivative, that match the true velocity and acceleration at every checkpoint. Therefore, the forcing used in the Navier-Stokes equations $(12)$ is now taken as

$$
\tilde{\mathbf{f}}(t)=-\lambda \omega_{f}(\mathbf{u}(t)-\tilde{\mathbf{u}}(t-T)) .
$$

In traditional check-pointing techniques, such as the one used in direct-adjoint optimization schemes (36, 37]), a new simulation is run from the checkpoint to avoid errors from interpolation. This strategy cannot be applied in the present case, due to endless interdependency between periods: the time-delayed feedback at $t-T$ requires the knowledge of the flow at $t-2 T$, which in turn depends on the flow state at $t-3 T$, and so forth.

The reconstruction technique has been evaluated for the paired jet case at $R e=2000$ and $S t=0.60$. Each period of the flow is composed of 1000 time steps, with $\Delta t=5 / 3 \times 10^{-3}$. Four cases have been investigated and compared to the results obtained without interpolation: $N=50$, 20, 10 and 5. These cases respectively need 10, 25, 50 and 100 times less memory than the fullstorage method (as memory is needed for the flow and its derivative).

In order to evaluate the convergence performance of the algorithm for various values of $N$, two residuals are used. The first one, denoted $\tilde{e}_{N}(t)$, is based on the interpolated velocity $\tilde{\mathbf{u}}$ at $t-T$ :

$$
\tilde{e}_{N}(t)=\frac{1}{2} \sqrt{\int_{z} \int_{r} r\|\mathbf{u}(t)-\tilde{\mathbf{u}}(t-T)\|^{2} \mathrm{~d} r \mathrm{~d} z} .
$$

The second one, denoted $e_{N}(t)$, is based on the true velocity $\mathbf{u}$ at $t-T$, as defined in equation (17). $\tilde{e}_{N}(t)$ is the only available residual when interpolation is applied in general, whereas $e_{N}(t)$ is the 


\begin{tabular}{c|cccc}
\hline \hline$N$ & 50 & 20 & 10 & 5 \\
\hline $\max _{t}\|\mathbf{u}(t)-\tilde{\mathbf{u}}(t)\|$ \\
\hline$e(T)$ & $3.6 \cdot 10^{-6}$ & $1.3 \cdot 10^{-4}$ & $1.5 \cdot 10^{-3}$ & $1.1 \times 10^{-2}$ \\
\hline \hline
\end{tabular}

TABLE I: Maximum normalized error between the interpolated and the real flow as a function of $N$ for a fully stabilized unpaired flow at $R e=2000$ and $S t=0.60$.

true residual, which is normally unknown. For each $N$, the evolution of each of these two residuals is compared to the evolution of the residual $e(t)$ obtained with the full-storage version (see Sec. IV B.

The convergence results with the interpolated residual $\tilde{e}_{N}(t)$ are depicted in Fig. 12 a. In every case, the residual first decreases in the same way as the uninterpolated stabilized flow. However, for $N<50$, the residual starts to oscillate at a critical residual threshold. These oscillations have a maximum peak value $\tilde{E}_{N}$, which depends on $N$, and they descend in all cases to the same residual level $E$ that is found in the full-storage solution (black line). The oscillation period corresponds to the interpolation period $T / N$. It is found that at the precise instants where snapshots are stored, the residual $\tilde{e}_{N}(t)$ is of the same order as the reference residual $E$.

In order to understand the meaning of this residual peak $\tilde{E}_{N}$, the maximum error between the interpolated and the real flow field as a function of $t$ and $N$ has been computed for the stabilized unpaired case. This maximum error occurs at $t=\left(t_{i}+t_{i+1}\right) / 2$ and is listed in Table I. For each $N$, the values obtained are of the same order as $\tilde{E}_{N}$ from Fig. 12 a. For $N=50$, the value $3.6 \cdot 10^{-6}$ is one order of magnitude smaller than $\min _{t} \tilde{e}_{50}(t)=6 \cdot 10^{-5}$, which explains why oscillations are not encountered in this case. The residual from the interpolated velocity $\tilde{e}_{N}(t)$ can then be understood as the sum of two components: the non- $T$-periodic component of the flow $e_{N}(t)$ and the interpolation error of the flow at $t-T$. At large times, the interpolation error component seems to dominate the interpolated residual $\tilde{e}_{N}(t)$. We will now prove this statement and show that interpolation does not affect the overall precision of the reconstructed flow.

For this, Fig. $12 \mathrm{~b}$ displays the evolution of the ratio between the residual $e_{N}(t)$ computed with the exact flow field for each interpolation level $N$ and the residual $e(t)$ from the full-storage reference case. For $t>30 T$, in the final phase of stabilization (see Fig. 12 ), the exact residual with interpolation $e_{N}(t)$ is only slightly above the residual from full-storage calculations. As $N$ increases, the interpolation improves and $e_{N}(t)$ approaches the reference value. It is found that the stabilized flow state obtained with checkpointing, even for $N=5$, is about as accurate as the full-storage solution, despite large residual values $\tilde{e}(t)$ between checkpoints. When interpolation is used and only $\tilde{e}_{N}(t)$ is available, the convergence of the algorithm should therefore be only assessed at times $t$ that corresponds to checkpoints at $t-T$.

\section{STABILIZATION OF LIMIT CYCLES UNKNOWN FREQUENCIES - THE LID DRIVEN CAVITY EXAMPLE}

When the frequency of the limit cycle is not known a priori, unlike the jet example, some techniques have been developed in the harmonic balance technique to overcome this issue, such as the Gradient-Based Variable Time Period 10 13. This technique is based on considering the residual as a function of not only $t$ but also $T$, and to choose $T$ as an extremum of this residual. This method, based in their case on gradient computations, can easily be transposed to our stabilization 


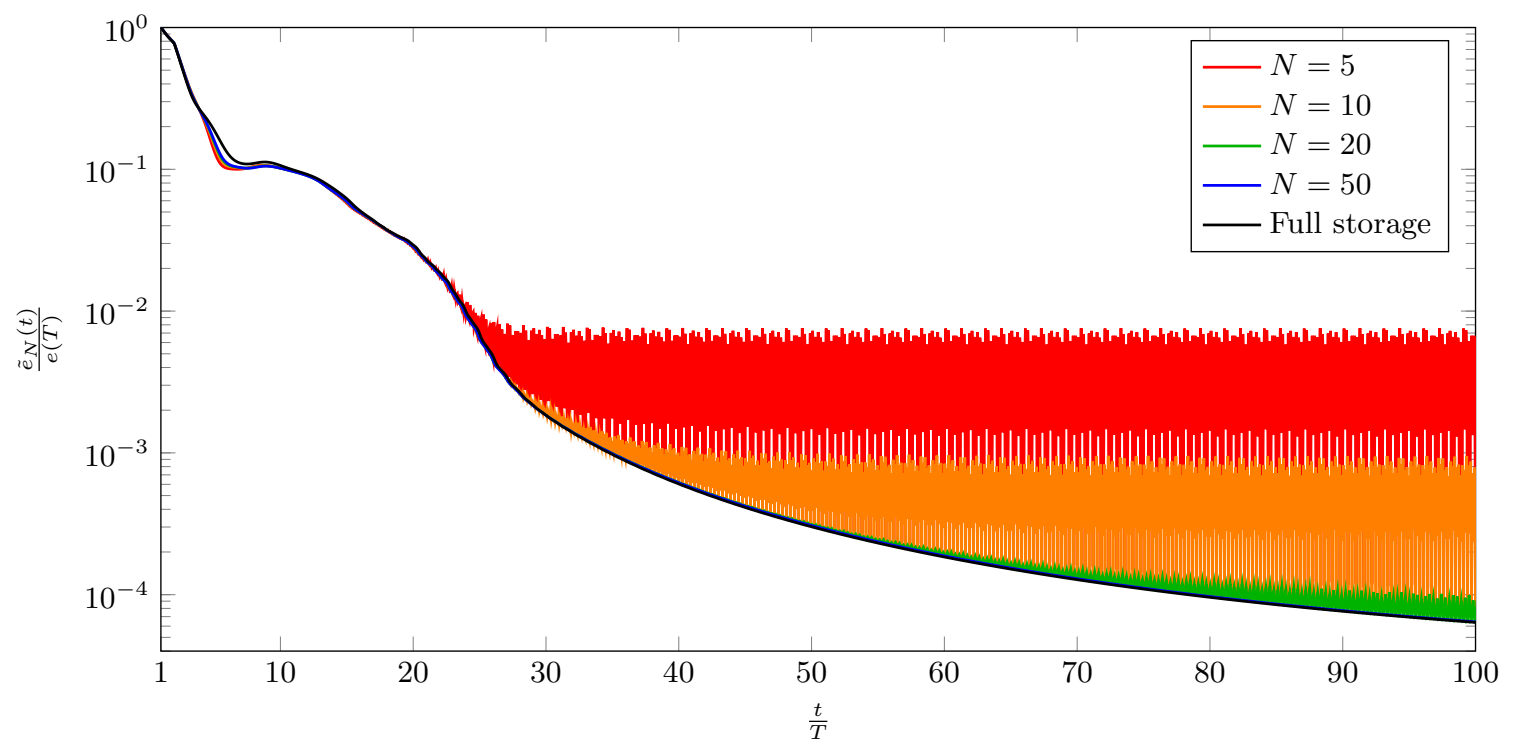

(a) Residual norm based on the interpolated velocity $\tilde{e}_{N}(t)$ as a function of time for different $N$ and comparison to full-storage residual $e(t)$.

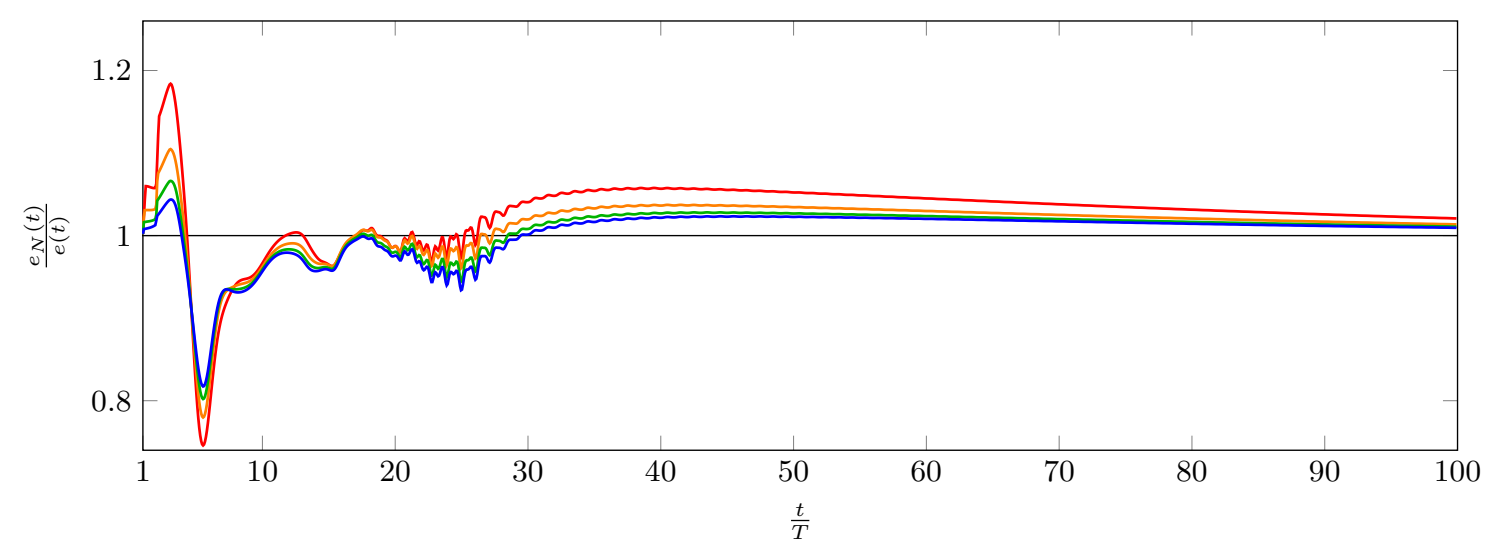

(b) Ratio between the exact residual $e_{N}(t)$ and the residual obtained with full storage $e(t)$ as a function of time for different $N$.

FIG. 12: Convergence analysis of the stabilization procedure with interpolation, for different storage requirements $N$. For $N=5,10$ and $20, \tilde{E}_{N}$ is defined as the maximum peak of $\tilde{e}_{N}(t)$ when the residual starts oscillating. For $N=50$, no oscillations are observed.

procedure:

- A starting guess $T_{g}$ of the period $T_{0}$ of the limit cycle is required.

- TDF is then applied with this $T_{g}$. Both the term $\mathbf{u}\left(t-T_{g}\right)$ and the dimensional $\lambda$ depend on $T_{g}$, see equation (16). 


\begin{tabular}{c|ccc}
\hline \hline & Feldman and Gelfgat $([26])$ & Kuhlmann and Albensoeder $([27])$ & Loiseau et al. ([28]) \\
\hline$R e_{c}$ & 1914.0 & 1919.5 & 1914.0 \\
$\omega_{c}$ & 0.575 & 0.586 & 0.585 \\
\hline \hline
\end{tabular}

TABLE II: Review of the critical Reynolds number and frequency of the linear unstable mode at $R e_{c}$ for the cubic lid-driven cavity.

- At $t=t_{1}$, when initial transients are stabilized, i.e. when $e\left(t_{1}, T_{g}\right)$ is small enough (for instance, $\left.e\left(t_{1}, T_{g}\right)<0.01\left\|\mathbf{u}\left(t_{1}\right)\right\|\right)$, a new value for $T_{g}$ is identified as the minimum

$$
T_{g}=\underset{T^{\prime} \in\left[0.8 T_{g} ; 1.2 T_{g}\right]}{\arg \min } e\left(t_{1}, T^{\prime}\right),
$$

with the residual $e(t, T)$ as defined in equation (17). This global search, almost inexpensive since $\mathbf{u}(t)$ and $\mathbf{u}\left(t-T^{\prime}\right)$ are already stored, is restricted to $\left[0.8 T_{g} ; 1.2 T_{g}\right]$ in order to avoid abrupt variations of $T_{g}$.

- The stabilization procedure is applied again with the new $T_{g}$ over a time-horizon equal to $T_{g}$.

- The global search is regularly carried out at $t_{i+1}=t_{i}+T_{g}$.

We prefer performing regular global searches for $T_{g}$ instead of calculating $\partial e / \partial T$, because the full storage of the past period allows to perform a cheap and quick optimization over a full range of $T_{g}$ values $\left(\left[0.8 T_{g} ; 1.2 T_{g}\right]\right)$ and because of the superior robustness provided by global methods compared to local methods.

As the limit-cycle frequency in the forced jet is prescribed by the applied forcing, it would be contrived to treat it as an unknown. The flow in a $3 D$ cubic lid-driven cavity is chosen instead for a demonstration. It has been shown that the steady solution of such a flow, above a critical Reynolds $R e_{c}$, is no longer stable [26 28, and that it bifurcates towards a limit cycle in a slightly subcritical fashion [27. The bifurcated state is unsteady and, close to $R e_{c}$, it evolves at the frequency $\omega_{c}$ predicted by linear stability theory. Critical Reynolds number and frequency are listed in Table II However, as shown in 27, 28, this limit cycle is not stable since it experiences intermittent chaos: short bursts occur that destabilize the cycle before disappearing. Therefore, without applying any stabilization technique, it cannot be expected that this cycle will converge naturally.

These simulations have been carried out again with NEK5000, on the same mesh as used in 28]. The driving velocity and the cube side length are used to non-dimensionalize the problem. A Reynolds number of 1930 - above the critical limit - is chosen. At this Reynolds number, the limitcycle frequency is kept unchanged at $\left.\omega_{0}=0.585(28]\right)$. The time step was fixed to $\Delta t=2.0 \cdot 10^{-3}$. In this study, all time steps have been stored (the method described in Sec. IV E was not applied). At $t=0$, the cavity is at rest: $\mathbf{u}(t=0)=\mathbf{0}$.

To understand the performance of the algorithm, several cases have been investigated:

- with no forcing,

- with forcing applied at the fixed frequency of the limit cycle, $\omega_{0}=0.585$.

- with variable-frequency forcing applied, starting form an initial guess. Five guess values have been tried: $\omega_{g}=0.50,0.55,0.60,0.65$, and 0.585 . The frequency interval covered is $\omega_{0} \pm 15 \%$. 


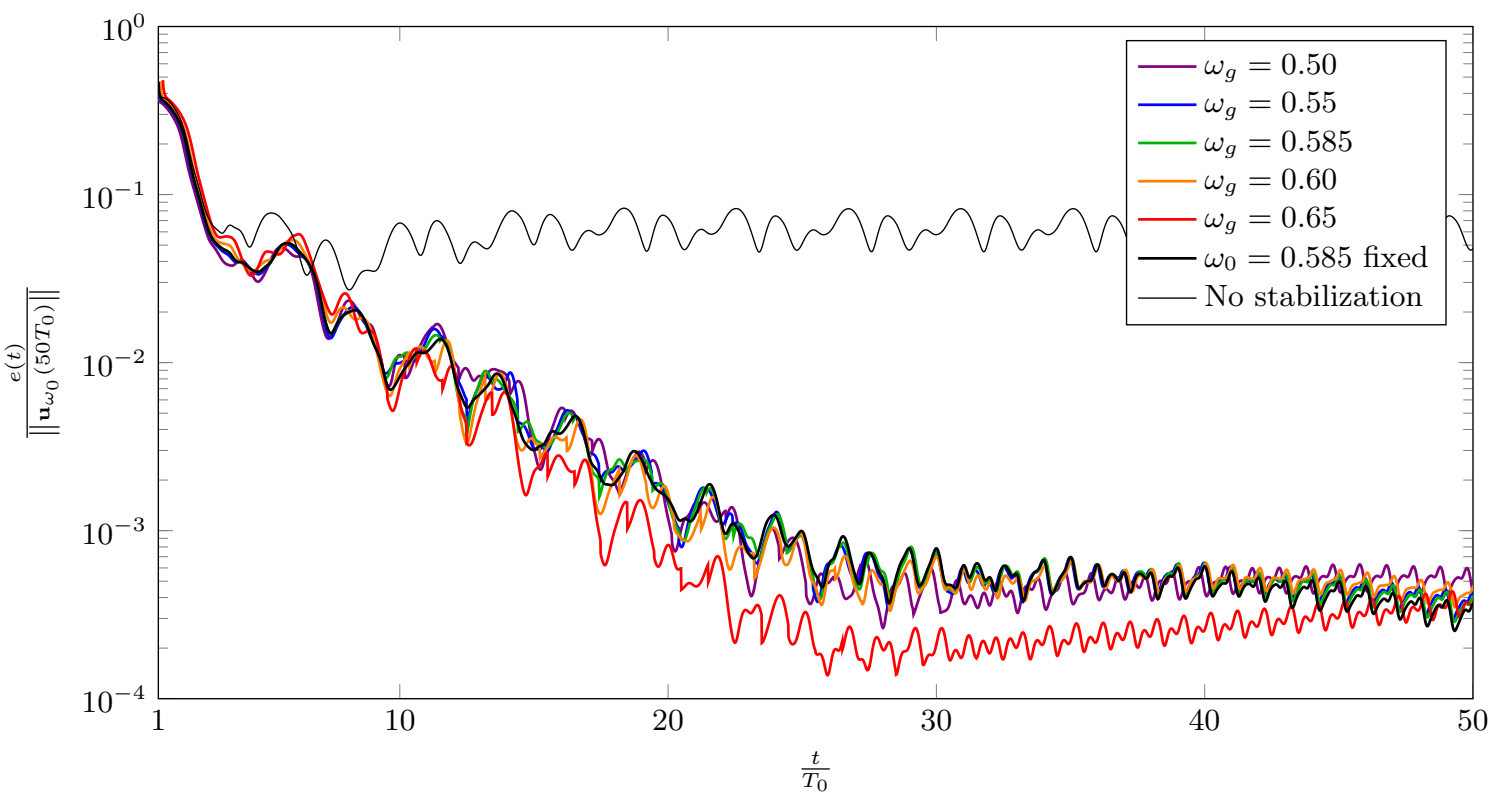

(a) Norm of the residual component as a function of time for the different cases studied normalized with the total velocity norm at $t=50 T_{0}$ for the fixed $\omega_{0}$ case.

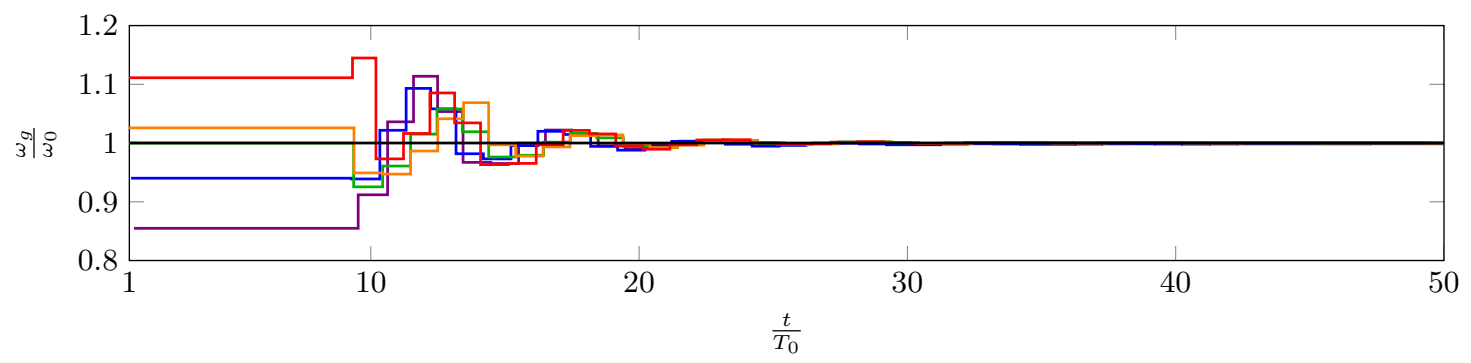

(b) Evolution of the global frequency guess for each case as a function of time.

FIG. 13: Convergence analysis of the lid-driven cavity.

The results are reported in Fig. 13. First, it can be stated that the method works for every $\omega_{g}$ studied: the convergence is improved by at least two orders of magnitude compared to the timestepping without stabilization. Moreover, the convergence of the flow and $\omega$ is achieved whatever $\omega_{g}$ studied, which shows the robustness of the technique. Convergence is achieved in about $25 T_{0}$ whatever $\omega_{g}$, which is the same physical time needed for the case with fixed $\omega_{0}$ to settle. Therefore, the frequency search does not augment significantly the computational cost. However, contrary to the unpaired jet, the decrease of the residual is not monotonic, which can be linked to the fact that the cavity flow is not receptive to $\omega_{0} / 2$ perturbations but to other frequencies [28]. 


\section{CONCLUSION}

A time-delayed feedback method, introduced by Pyragas 23 in the context of ODEs with few degrees of freedom, has been applied to a flow problem for the purpose of computing unstable time-periodic states. It has been demonstrated that spontaneous vortex pairing in a harmonically forced jet is efficiently suppressed by this method, such that an unpaired vortex street, synchronized at the frequency of the prescribed inflow forcing, is recovered. In this final converged flow state, the stabilizing feedback term vanishes, and the recovered state is therefore a true solution of the flow equations, uncompromised by artificial damping. The one free numerical parameter for this procedure has been chosen based on a simple model problem, where the optimal value could be determined analytically. It has then been found that the same value provides optimal convergence also in the jet calculations.

The same technique has been shown to be useful also in weakly stable situations, where uncontrolled time-stepping converges towards a T-periodic state, but only slowly so. Artificial damping through time-delay feedback greatly increases the rate of convergence in this case.

The described method is very easy to implement with a given flow solver, as it only requires the addition of a simple source term, as well as the storage of one full cycle of the flow. The latter aspect may be memory resource-intensive. An interpolation method has been proposed in order to overcome this limitation. In the jet example, the storage requirement could thus be reduced by a factor 100, without significant loss of accuracy, and at negligible additional cost.

The suppression of vortex pairing in the present example enables a stability analysis of the recovered unpaired state, and the results of such analysis will be reported in a forthcoming study.

The time-delayed feedback method has finally been adapted to stabilize limit cycles in unforced flows, where the frequency is not known a priori. This was demonstrated for a lid-driven cubic cavity case with intermittent chaos. The procedure has been found to be very effective, enabling limit-cycle stabilization at the correct frequency. The iteration identification of the limit-cycle frequency, as an additional unknown, did not lead to prolonged simulations in the cavity example. As in the harmonically forced jet, the recovered state is a true solution of the flow equations.

\section{ACKNOWLEDGEMENTS}

We gratefully acknowledge valuable discussions with Olivier Marquet. We thank Holly Johnson for her help with NEK5000 and Jean-Christophe Loiseau for suggesting the lid-driven cubic cavity as a test case and for providing his NEK5000 mesh.

This work was granted access to the HPC resources of TGCC under the allocation 2016-2a6451 made by GENCI, and it benefited from financial support of the DGA under grant 2015.60.0004.

\section{Appendix: Stability of solutions to equations (9a) and (9c)}

Consider the equation

$$
\alpha=k i-\lambda\left(1-e^{-2 \pi \alpha}\right),
$$

with both $k$ and $\lambda$ having positive real values. Solutions [33] are found as

$$
\alpha_{j}=k i-\lambda+\frac{1}{2 \pi} W_{j}\left(2 \pi \lambda e^{2 \pi(\lambda-i k)}\right), \quad j \in \mathbb{Z} .
$$


The $j^{\text {th }}$ solution involves the $j^{\text {th }}$ branch $W_{j}$ of the Lambert function. Assuming that there exists a branch $W_{j}$ such that $\operatorname{Re}\left(\alpha_{j}\right)>\beta$ for a given $\lambda$, the triangular inequality, applied to equation (A.1), guarantees

$$
\left|\alpha_{j}\right| \leq|k i|+\lambda\left|1-e^{-2 \pi \alpha_{j}}\right| \leq k+\left(1+e^{-2 \pi \beta}\right) \lambda .
$$

The imaginary part of $(\mathrm{A} .2 \mathrm{n}$ is evaluated as

$$
\operatorname{Im}\left(\alpha_{j}\right)=k+\frac{1}{2 \pi} \operatorname{Im}\left[W_{j}\left(2 \pi \lambda e^{2 \pi(\lambda-i k)}\right)\right] .
$$

Positive and negative integer values of $j$ need to be considered separately.

\section{Case $j>0$}

In this case, from [33], as $\operatorname{Im}\left(W_{j}(z)\right)>0$ for all complex number $z$ and $k>0$ :

$$
\left|\operatorname{Im}\left(\alpha_{j}\right)\right|=k+\frac{1}{2 \pi} \operatorname{Im}\left[W_{j}\left(2 \pi \lambda e^{2 \pi(\lambda-i k)}\right)\right],
$$

so that, as $\left|\alpha_{j}\right| \geq\left|\operatorname{Im}\left(\alpha_{j}\right)\right|$ :

$$
\left|\alpha_{j}\right| \geq k+\frac{1}{2 \pi} \operatorname{Im}\left[W_{j}\left(2 \pi \lambda e^{2 \pi(\lambda-i k)}\right)\right] .
$$

Therefore, combining (A.3) and A.6):

$$
\operatorname{Im}\left[W_{j}\left(2 \pi \lambda e^{2 \pi(\lambda-i k)}\right)\right] \leq 2 \pi\left(1+e^{-2 \pi \beta}\right) \lambda .
$$

From the properties of the Lambert function [33, and because $j>0, \operatorname{Im}\left(W_{j}(z)\right)>2 \pi(j-1)$ for all complex $z$. Therefore a necessary condition for $\operatorname{Re}\left(\alpha_{j}\right)>\beta$ with $j>0$ is:

$$
|j|<1+\left(1+e^{-2 \pi \beta}\right) \lambda .
$$

\section{Case $j<0$}

In this case, from [33, as $\operatorname{Im}\left(W_{j}(z)\right)<0$ for all complex number $z$ and $k>0$ :

$$
\left|\operatorname{Im}\left(\alpha_{j}\right)\right|=k-\frac{1}{2 \pi} \operatorname{Im}\left[W_{j}\left(2 \pi \lambda e^{2 \pi(\lambda-i k)}\right)\right],
$$

so that, as $\left|\alpha_{j}\right| \geq\left|\operatorname{Im}\left(\alpha_{j}\right)\right|$ :

$$
\left|\alpha_{j}\right| \geq k-\frac{1}{2 \pi} \operatorname{Im}\left[W_{j}\left(2 \pi \lambda e^{2 \pi(\lambda-i k)}\right)\right] .
$$

Therefore, combining (A.3) and A.10):

$$
-\operatorname{Im}\left[W_{j}\left(2 \pi \lambda e^{2 \pi(\lambda-i k)}\right)\right] \leq 2 \pi\left(1+e^{-2 \pi \beta}\right) \lambda .
$$

From the properties of the Lambert function [33], and because $j<0, \operatorname{Im}\left(W_{j}(z)\right)<2 \pi(j+1)$ for all complex $z$. Therefore a necessary condition for $\operatorname{Re}\left(\alpha_{j}\right)>\beta$ with $j<0$ is:

$$
|j|<1+\left(1+e^{-2 \pi \beta}\right) \lambda .
$$




\section{Conclusion}

The two cases $j \lessgtr 0$ leads to the same conclusion, which is also valid for $j=0$. Therefore, for a given $\lambda$, any mode $\alpha_{j}$ such that $\operatorname{Re}\left(\alpha_{j}\right)>\beta$ must derive from branches $W_{j}$ with:

$$
|j|<1+\left(1+e^{-2 \pi \beta}\right) \lambda .
$$

This criterion is strict and holds for any value of $k$.

In particular, for a given $\lambda$, the unstable modes, if they exist, must derive from branches $W_{j}$ with $|j|<1+2 \lambda$.

[1] Denis Sipp and Anton Lebedev, "Global stability of base and mean flows: a general approach and its applications to cylinder and open cavity flows," Journal of Fluid Mechanics 593, 333-358 (2007).

[2] Gautam M. Shroff and Herbert B. Keller, "Stabilization of unstable procedures: the recursive projection method," SIAM Journal on numerical analysis 30, 1099-1120 (1993).

[3] Michele Sergio Campobasso and Michael B. Giles, "Stabilization of a linear flow solver for turbomachinery aeroelasticity using recursive projection method," AIAA journal 42, 1765-1774 (2004).

[4] Espen Åkervik, Luca Brandt, Dan S. Henningson, Jérôme Hœpffner, Olaf Marxen, and Philipp Schlatter, "Steady solutions of the Navier-Stokes equations by selective frequency damping," Physics of Fluids (1994-present) 18, 068102 (2006).

[5] Ronald D. Henderson and Dwight Barkley, "Secondary instability in the wake of a circular cylinder," Physics of Fluids 8, 1683-1685 (1996).

[6] S. J. Sherwin and Hugh M. Blackburn, "Three-dimensional instabilities and transition of steady and pulsatile axisymmetric stenotic flows," Journal of Fluid Mechanics 533, 297-327 (2005).

[7] Frédéric Sicot, Guillaume Dufour, and Nicolas Gourdain, "A time-domain harmonic balance method for rotor/stator interactions," Journal of Turbomachinery 134, 011001 (2012).

[8] Kenneth C. Hall, Jeffrey P. Thomas, and William S. Clark, "Computation of unsteady nonlinear flows in cascades using a harmonic balance technique," AIAA journal 40, 879-886 (2002).

[9] Jeffrey P. Thomas, Earl H. Dowell, and Kenneth C. Hall, "Nonlinear inviscid aerodynamic effects on transonic divergence, flutter, and limit-cycle oscillations," AIAA journal 40, 638-646 (2002).

[10] Matthew McMullen, Antony Jameson, and Juan J. Alonso, "Application of a non-linear frequency domain solver to the Euler and Navier-Stokes equations," AIAA paper 13625 (2002).

[11] Meredith A. Spiker, Jeffrey P. Thomas, R. E. Kielb, K. C. Hall, and Earl H. Dowell, "Modeling cylinder flow vortex shedding with enforced motion using a harmonic balance approach," AIAA Paper 1965, 2006 (2006).

[12] Arathi Gopinath and Antony Jameson, "Application of the time spectral method to periodic unsteady vortex shedding," AIAA paper 449 (2006).

[13] Matthew McMullen, Antony Jameson, and Juan Alonso, "Demonstration of nonlinear frequency domain methods," AIAA journal 44, 1428-1435 (2006).

[14] Kivanc Ekici and Kenneth C. Hall, "Nonlinear frequency-domain analysis of unsteady flows in turbomachinery with multiple excitation frequencies," AIAA journal 46, 1912-1920 (2008).

[15] S. K. Nadarajah, M. S. McMullen, and A. Jameson, "Optimum shape design for unsteady flow using time accurate and nonlinear frequency domain methods," AIAA Paper 3875 (2003).

[16] Siva Nadarajah and Antony Jameson, "Optimum shape design for unsteady three-dimensional viscous flows using a nonlinear frequency-domain method," Journal of Aircraft 44, 1513-1527 (2007).

[17] K. B. M. Q. Zaman and A. K. M. F. Hussain, "Vortex pairing in a circular jet under controlled excitation. Part 1. General jet response," Journal of Fluid Mechanics 101, 449-491 (1980).

[18] Baptiste Bourget, Thierry Dauxois, Sylvain Joubaud, and Philippe Odier, "Experimental study of 
parametric subharmonic instability for internal plane waves," Journal of Fluid Mechanics 723, 1-20 (2013).

[19] Dirk Roose, Kurt Lust, A. Champneys, and A. Spence, "A Newton-Picard shooting method for computing periodic solutions of large-scale dynamical systems," Chaos, Solitons \& Fractals 5, 19131925 (1995).

[20] Kurt Lust and Dirk Roose, "An adaptive Newton-Picard algorithm with subspace iteration for computing periodic solutions," SIAM Journal on Scientific Computing 19, 1188-1209 (1998).

[21] J. Sánchez, M. Net, B. Garcia-Archilla, and C. Simó, "Newton-Krylov continuation of periodic orbits for Navier-Stokes flows," Journal of Computational Physics 201, 13-33 (2004).

[22] Juan Sánchez and Marta Net, "On the multiple shooting continuation of periodic orbits by NewtonKrylov methods," International Journal of Bifurcation and Chaos 20, 43-61 (2010).

[23] Kestutis Pyragas, "Continuous control of chaos by self-controlling feedback," Physics Letters A 170, 421-428 (1992).

[24] Damien Jallas, Olivier Marquet, and David Fabre, "Linear and nonlinear perturbation analysis of the symmetry breaking in time-periodic propulsive wakes," Physical Review E 95, 063111 (2017).

[25] PN Shankar and MD Deshpande, "Fluid mechanics in the driven cavity," Annual Review of Fluid Mechanics 32, 93-136 (2000).

[26] Yuri Feldman and Alexander Yu. Gelfgat, "Oscillatory instability of a three-dimensional lid-driven flow in a cube," Physics of Fluids 22, 093602 (2010).

[27] Hendrik C. Kuhlmann and Stefan Albensoeder, "Stability of the steady three-dimensional lid-driven flow in a cube and the supercritical flow dynamics," Physics of Fluids 26, 024104 (2014).

[28] Jean-Christophe Loiseau, Jean-Christophe Robinet, and Emmanuel Leriche, "Intermittency and transition to chaos in the cubical lid-driven cavity flow," Fluid Dynamics Research 48 (2016).

[29] Chih-Ming Ho and Patrick Huerre, "Perturbed free shear layers," Annual Review of Fluid Mechanics 16, 365-422 (1984).

[30] Karl Johan Åström and Richard M. Murray, Feedback systems: an introduction for scientists and engineers (Princeton university press, 2008).

[31] John C. Doyle, Bruce A. Francis, and Allen R. Tannenbaum, Feedback control theory (Macmillan Publishing Company, 1992).

[32] Wim Michiels and Silviu-Iulian Niculescu, Stability and Stabilization of Time-Delay Systems (Advances in Design 83 Control) (Advances in Design and Control) (Society for Industrial and Applied Mathematics, Philadelphia, PA, USA, 2007).

[33] R. M. Corless, G. H. Gonnet, D. E. G. Hare, D. J. Jeffrey, and D. E. Knuth, "On the Lambert W function," Advances in Computational Mathematics 5, 329-359 (1996)

[34] Paul F. Fischer, James W. Lottes, and Stefan G. Kerkemeier, "Nek5000 web page," NEK5000 - A spectral element code for CFD (2008).

[35] R. G. Jacobs and P. A. Durbin, "Simulations of bypass transition," Journal of Fluid Mechanics 428, 185-212 (2001).

[36] Peter J Schmid, "Nonmodal stability theory," Annu. Rev. Fluid Mech. 39, 129-162 (2007).

[37] Michael Hinze, Andrea Walther, and Julia Sternberg, "An optimal memory-reduced procedure for calculating adjoints of the instationary navier-stokes equations," Optimal Control Applications and Methods 27, 19-40 (2006). 